\title{
EMBARAZO DE GEMELO ACÁRDICO COEXISTENTE CON GEMELO ANENCEFÁLICO, ASOCIADO A EXPOSICIÓN A VARICELA EN EL PRIMER TRIMESTRE: REPORTE DE CASO Y REVISIÓN DE LA LITERATURA
}

\author{
A case report and literature review regarding \\ acardiac twin pregnancy coexisting with \\ anencephalic twin, associated with being exposed \\ to chicken pox during the first trimester of \\ preginancy \\ Wilmar Saldarriaga-Gil, M.D., M.Sc. *, Álbaro José Nieto-Calvache, M.D.**, \\ Harry Pachajoa, M.D.***, Katherine Tabares, M.D.****, \\ Carolina Isaza, M.D., M.Sc. ***** \\ Recibido: marzo 9/10 - Aceptado: noviembre 12/10
}

\section{RESUMEN}

Introducción: el gemelo acárdico es una malformación congénita de muy baja prevalencia, 1 en 35.000 nacimientos, y se encuentra aproximadamente en 1 de cada 100 gemelos

* Gineco-obstetra. Ciencias Básicas Médicas, Énfasis Embriología y Genética. MACOS, Grupo de Malformaciones Congénitas Perinatales y Dismorfología, Universidad del Valle. Profesor Asistente. Departamentos: Morfología, Ginecología y Obstetricia, Facultad de Salud, Universidad del Valle. Ginecólogo, Hospital Universitario del Valle y Fundación Valle del Lili. Cali (Colombia). Correo electrónico: wsaldarriaga0608@yahoo.com

** Jefe de residentes, Departamento de Ginecología y Obstetricia, Universidad del Valle. MACOS, Grupo de Malformaciones Congénitas Perinatales y Dismorfología, Universidad del Valle. Cali (Colombia).

*** Candidato a Doctor en Ciencias Biomédicas. Universidad del Valle. Profesor Asistente. Departamento de Morfología, Facultad de Salud, Universidad del Valle. Facultad de Medicina, Universidad Icesi. MACOS, Grupo de Malformaciones Congénitas Perinatales y Dismorfología, Universidad del Valle. Cali (Colombia).

**** Gineco-obstetra. Universidad del Valle. MACOS, Grupo de Malformaciones Congénitas Perinatales y Dismorfología, Universidad del Valle. Cali (Colombia).

****** Profesora Titular, Departamento de Morfología, Facultad de Salud, Universidad del Valle. Vicerrectora de Investigaciones, Universidad del Valle. MACOS, Grupo de Malformaciones Congénitas Perinatales y Dismorfología, Universidad del Valle. Cali (Colombia). monocigóticos. La asociación de un gemelo acárdico con un segundo gemelo con malformaciones mayores ocurre aproximadamente en el 10\% de los casos. El objetivo de este artículo es el reporte de un caso de muy baja prevalencia y la revisión de la literatura publicada sobre la etiología y fisiopatología asociada a la acardia, así como de las anomalías congénitas asociadas a esta y a la infección por virus de la varicela.

Materiales y métodos: se presenta el caso de un embarazo gemelar monocoriónico biamniótico con un gemelo acardius mylacephalus y un segundo gemelo anencefálico, con exposición de la madre a varicela en el primer trimestre del embarazo. Se realizó cariotipo con bandas G, 46 XY sin anormalidades estructurales. Se hace una revisión de la literatura publicada en los últimos 10 años en las bases de datos PubMed, Ovid e HINARI.

Conclusión: la importancia de este trabajo radica en que en la literatura revisada sólo se 
encontró un único reporte de la asociación de gemelo arcádico, coexistente con gemelo anencefálico. Además, sería el primer reporte de la relación entre esta combinación de malformaciones mayores en el embarazo gemelar y la exposición a varicela en el primer trimestre del embarazo.

Palabras clave: gemelo acárdico, anencefalia, varicela, ECLAMC.

\section{SUMMARY}

Introduction: an acardiac twin is a congenital malformation having low prevalence (1 in $35,000)$ births and is found in about 1 in every 100 monozygotic twins. The association of an acardiac twin with a second twin having greater malformations occurs in around 10\% of cases. This article was aimed at reporting a case having very low prevalence and a review of the literature published about the etiology and physiopathology associated with acardiac anomaly, as well as the congenital anomalies associated with it and infection by the chicken pox virus.

Materials and methods: the case of monochorionicdiamniotic twin pregnancy involving an acardius mylacephalus twin and a second anencephalic twin is presented, the mother having been exposed to chicken pox during the first trimester of pregnancy. G-band kariotyping showed 46 $\mathrm{XY}$ having no structural abnormalities. A search was made of the literature published during the last 10 years in the PubMed, Ovid and HINARI databases.

Conclusion: the importance of this report lies in the fact that only one report of the association of acardiac twin coexisting with anencephalic twin was found in the pertinent literature. This is also the first report of the relationship between this combination of greater malformations in twin pregnancy and exposure to chicken pox during the first trimester of pregnancy.

Key words: acardiac twin, anencephaly, chicken pox, ECLAMC.

\section{INTRODUCCIÓN}

La acardia es una malformación congénita definida por la ausencia del desarrollo del corazón y porciones variables del cuerpo fetal, en la cual se implica la secuencia de perfusión arterial reversa gemelar. La frecuencia de presentación es de 1 en 35.000 nacimientos y se encuentra aproximadamente en 1 de cada 100 gemelos monocigóticos. ${ }^{1}$ En el presente artículo se expone un caso de un gemelo con acardia asociado a la presencia de anencefalia en el segundo gemelo y la exposición previa a infección por varicela en el embarazo, con el objetivo de hacer una revisión de la literatura publicada al respecto, de la etiología de esta malformación congénita y de la fisiopatología asociada a la acardia. También se revisa la fisiopatología de las malformaciones congénitas en la infección por virus de la varicela. El caso aquí reportado fue detectado a través del sistema de vigilancia epidemiológica de malformaciones congénitas que se lleva a cabo en el Hospital Universitario del Valle, bajo la metodología del Estudio Colaborativo Latinoamericano de Malformaciones Congénitas (ECLAMC).

\section{REPORTE DE CASO}

Se reporta un embarazo gemelar monocoriónico biamniótico en mujer de 28 años en su segunda gestación, quien presentó un parto pretérmino a las 28 semanas de gestación. Dentro de los antecedentes del embarazo afirmó haber presentado, entre las 6 y 7 semanas de embarazo, vesículas en todo el cuerpo asociadas a fiebre, los síntomas fueron interpretados por su médico como varicela y fue manejada con acetaminofén. Asistió a 3 controles prenatales. En la semana 18, le fue practicada una ecografía que mostró un gemelo acárdico y el otro anencefálico. Al nacimiento, el gemelo número 1, acárdico de $540 \mathrm{~g}$, sin signos de vida, se presentó como una masa amorfa, de consistencia blanda, hidrópico; como estructuras definidas sólo se identificaron un miembro inferior y un esbozo de extremidad superior, sin poder definir su lateralidad, el tronco y cabeza no se encontraban formados (figura 1). 
En el gemelo número 2, con 500 g, como hallazgos patológicos, se encontró anencefalia y genitales externos indiferenciados (figura 2). Se realizó cariotipo con bandeo $\mathrm{G}$ a una resolución de 800 bandas de sangre obtenida por cordocentesis de ambos fetos, tras lo cual se encontró un complemento cromosómico 46 XY.

Figura 1. Gemelo acárdico, tipo acardius mylacephalus, de 540 g. Se observa ausencia de cabeza y esbozo de extremidad superior, un único miembro inferior, sin poder definir su lateralidad.

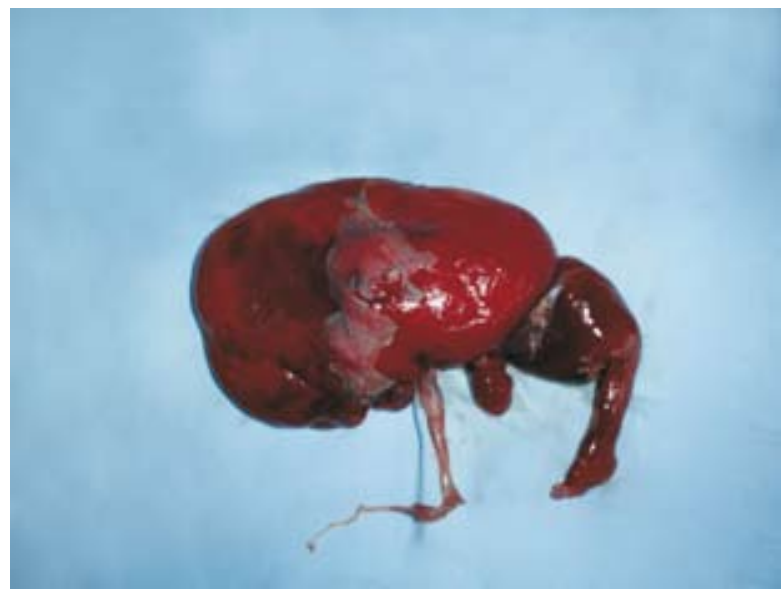

Figura 2. Gemelo anencefálico de 500 g. Se observó además genitales externos indiferenciados

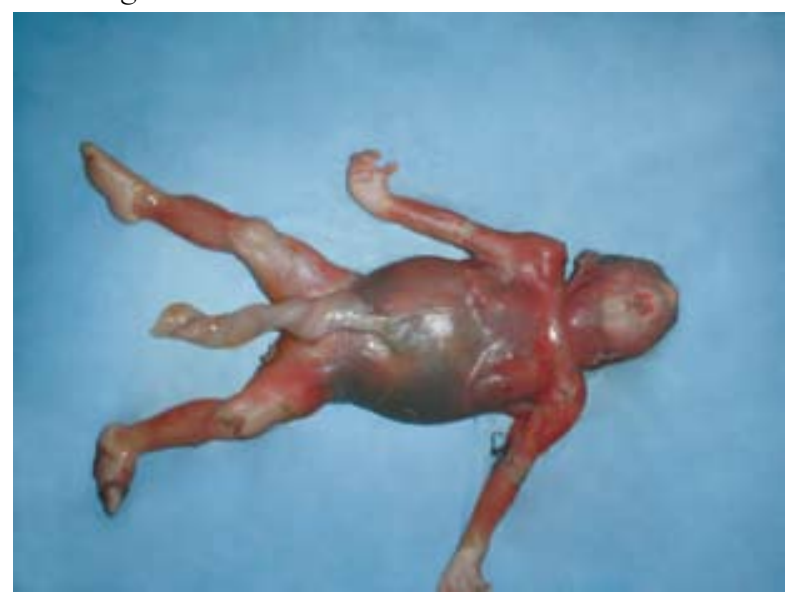

\section{MATERIALES Y MÉTODOS}

Se realizó una búsqueda sistemática de la literatura publicada en las bases de datos PubMed, Ovid e HINARI, utilizando las palabras clave: "acardiac twin", "anencephaly", "varicella/chicken pox", "ECLAMC"; especialmente, se buscaron reportes y series de casos. Como resultado de la búsqueda se encontró un único reporte sobre gemelo acárdico, coexistente con gemelo anencefálico. No se hallaron informes previos de la asociación entre esta combinación de malformaciones mayores en el embarazo gemelar y la exposición a varicela en el primer trimestre del embarazo.

\section{DISCUSIÓN}

La primera hipótesis en la patogénesis del gemelo acárdico sugería una deficiencia primaria en la formación de capas germinales, con la consecuente alteración en el desarrollo del corazón fetal. Según esta hipótesis, el gemelo acárdico se mantendría mediante anastomosis entre vasos de su cordón umbilical y la circulación del gemelo "bomba". ${ }^{2}$ A través de estudios ecográficos ${ }^{3}$ se ha demostrado flujo reverso por la vena umbilical del feto acárdico, implicándose este fenómeno como el factor etiológico en el daño del tejido cardíaco en formación, en lugar de que ocurra una agenesia cardíaca. La aplicación del concepto de secuencia de perfusión arterial reversa gemelar (twin reversed arterial perfusion, TRAP), enfatiza en el rol primario de las anastomosis arteria-arteria y vena-vena en la placenta, en la reversión del flujo sanguíneo de un gemelo al otro. Parece que esas anastomosis, en la placentación monocorial, ocurren en el primer trimestre del embarazo. La estrecha proximidad de las arterias umbilicales en desarrollo puede contribuir al establecimiento de estas anastomosis. El flujo reverso puede ocurrir en cualquiera de los gemelos, de esta manera el gemelo con ventaja hemodinámica se convierte en el gemelo "bomba" y el perfundido, de manera retrógrada, se convierte en el acárdico.

En el gemelo acárdico, el corazón, cerebro y otros órganos fallan en desarrollarse o sufren regresión debido a la circulación aberrante. Es frecuente que se presente piel, cabello, huesos largos, vértebras y colon; con menos frecuencia se encuentran hígado, tiroides y pulmones. La clave diagnóstica es 
la ausencia o formación rudimentaria del corazón. La cabeza puede estar ausente (acardius acephalus) o pobremente diferenciada en relación con el cuerpo (acardius anceps); en otras ocasiones, la cabeza es el único segmento desarrollado (acardius acomus). En la forma extrema del desorden, el gemelo acárdico se presenta como una masa de tejido amorfo sin diferenciación de órganos (acardius amorphous). Cuando en esta masa amorfa se diferencia un grado variable de desarrollo de extremidades se denomina acardius mylacephalus. ${ }^{2}$

El gemelo "bomba", por lo general, es anatómicamente normal, pero suele presentar falla cardíaca congestiva, dada la sobrecarga de volumen como consecuencia de la necesidad de perfundir el gemelo acárdico. Sin embargo, presenta malformaciones mayores en aproximadamente el 10\% de los casos; ${ }^{4,5}$ se encuentran reportes de gastrosquisis, atresia colónica, atresia del apéndice cecal, ${ }^{6}$ síndrome de Prune Belly, ${ }^{7}$ transposición de grandes vasos, ${ }^{8}$ necrosis cortical renal, asociación de VATER, ventrículo único ${ }^{9}$ y defectos del sistema nervioso central como necrosis cerebelar, hidranencefalia, porencefalia y microcefalia. ${ }^{5}$ Algunas de estas pueden ser explicadas por la teoría de disrupción vascular, disminución de la irrigación en una estructura formada adecuadamente. ${ }^{4}$ Además, se han reportado anormalidades citogenéticas como $47 \mathrm{XXY} .^{10,11}$

En nuestro reporte, los hallazgos del gemelo acárdico caben dentro de la descripción de acardius mylacephalus, descrito por Simonds y Gowen, ${ }^{11}$ que debe ser diferenciada del subtipo acardius acephalus. ${ }^{6,12-14}$ La coexistencia de gemelo acárdico con un gemelo "bomba" con anencefalia fue informada por Pavone en 1985, ${ }^{15}$ constituyéndose en una muy rara combinación de malformaciones.

Por otra parte, la infección por varicela en mujeres embarazadas con edad gestacional menor a 20 semanas se ha relacionado con embriopatía en el $0,78 \%{ }^{16}$ al 3\% de los casos. ${ }^{17}$ En fetos afectados, se han observado lesiones cutáneas en extremidades hipoplásicas y, en algunos casos, se llega a comprometer el tronco. Al parecer, las anormalidades en las extremidades se deben probablemente a neuropatía por lesiones de ganglios dorsales de la columna anterior de la médula espinal. ${ }^{14,16}$ La patología del sistema nervioso central es común e incluye microcefalia, atrofia cortical y cerebelosa, retardo psicomotor, convulsiones y calcificaciones focales del cerebro. Algunos recién nacidos afectados tienen disfunción del sistema nervioso autónomo manifestada por pérdida del control de esfínteres, disfagia, obstrucción intestinal o síndrome de Horner. ${ }^{16,17}$ En la literatura revisada no se encontraron reportes de la relación entre exposición a varicela en el primer trimestre del embarazo y embarazo gemelar, con un producto acárdico y el otro gemelo con malformaciones.

La importancia de este caso radica en que sería el primer reporte de un embarazo gemelar monocoriónico biamniótico con un gemelo acardius mylacephalus y un gemelo "bomba" anencefálico, con infección de la madre por varicela en el primer trimestre del embarazo. Además, se hace énfasis en el hecho de que al diagnosticarse un gemelo acárdico se debe realizar una ecografía de detalle de la anatomía del gemelo "bomba".

\section{CONSIDERACIONES ÉTICAS}

El reporte del caso y la publicación de las fotografías se hacen con la firma del consentimiento informado de los padres. El sistema de vigilancia de malformaciones congénitas en el Hospital Universitario del Valle que utiliza la metodología ECLAMC a través del cual se detectó el caso aquí reportado no tiene financiación. Además aclaramos que no existen conflictos de interés en el presente reporte.

\section{AGRADECIMIENTOS}

A todos los colaboradores de la vigilancia epidemiológica de malformaciones congénitas en el Hospital Universitario del Valle que utiliza la metodología ECLAMC, y a los integrantes del grupo de investigación MACOS (Malformaciones Congénitas Perinatales y Dismorfología). 


\section{REFERENCIAS}

1. Van Allen MI, Smith DW, Shepard TH. Twin reversed arterial perfusion (TRAP) sequence: a study of 14 twin pregnancies with acardius. Semin Perinatol 1983;7:285-93.

2. Hanafy A, Peterson CM. Twin-reversed arterial perfusion (TRAP) sequence: case reports and review of literature. Aust N Z J Obstet Gynaecol 1997;37:18791.

3. Coulam CB, Wright G. First trimester diagnosis of acardiac twins. Early Pregnancy 2000;4:261-70.

4. Schinzel AA, Smith DW, Miller JR. Monozygotic twinning and structural defects. J Pediatr 1979;95:921-30.

5. Moore TR, Gale S, Benirschke K. Perinatal outcome of forty-nine pregnancies complicated by acardiac twinning. Am J Obstet Gynecol 1990;163:907-12.

6. Habbal OA, Kenue RK, Venugopalan P. Acardia syndrome coexisting with gastroschisis in the co-twin. Clin Dysmorphol 2005;14:45-7.

7. Buntinx IM, Bourgeois N, Buytaert PM, Dumon JE. Acardiac amorphous twin with prune belly sequence in the co-twin. Am J Med Genet 1991;39:453-7.

8. Ustüner I, Simşek E, Kahraman K, Cengiz B, Koç A. Acardiac acephalic twin gestation with transposition of great arteries in pump twin. Congenit Anom (Kyoto) 2005;45:70-2.

9. Mühlhaus K, Behrens O, Degenhardt F. Antenatal diagnosis of a rare combined fetal malformation: acardius acephalus and single ventricle of the twin. Am J Perinatol 1991;8:251-4.

10. Moore CA, Buehler BA, McManus BM, Harmon JP, Mirkin LD, Goldstein DJ. Acephalus-acardia in twins with aneuploidy. Am J Med Genet Suppl 1987;3:13943.

11. Simonds JP. Fetus amorphous: report of a case. Surg Gynecol Obstet 1925;41:171-5.

12. Chanoufi MB, Ben Temime R, Masmoudi A, Ounaïssa K, Jebnoun S, Abid W, et al. Clinical and anatomic features of acardiac twins. Med Princ Pract 2004;13:375-9.

13. Chmait R, Hull A. Placental pathology casebook. Unusually large acardiac twin pregnancy at term. J Perinatol 2001;21:150-2.

14. Emery SC, Vaux KK, Pretorius D, Masliah E, Benirschke K. Acardiac twin with externalized intestine adherent to placenta: unusual manifestation of omphalocele. Paediatr Dev Pathol 2004;7:81-5

15. Pavone L, Laurence KM, Mattina T, Nuciforo G, Mollica F. Twins with acardia and anencephaly. Acta Genet Med Gemellol (Roma) 1985;34:89-93.

16. Koren G. Risk of varicella infection during late pregnancy. Can Fam Physician 2003;49:1445-6.

17. Balducci J, Rodis JF, Rosengren S, Vintzileos AM, Spivey G, Vosseller C. Pregnancy outcome following first-trimester varicella infection. Obstet Gynecol 1992;79:5-6. 